\title{
Laser power stabilization using optical ac coupling and its quantum and technical limits
}

\author{
Patrick Kwee, ${ }^{*}$ Benno Willke, and Karsten Danzmann \\ Max-Planck-Institut für Gravitationsphysik (Albert-Einstein-Institut) \\ and Leibniz Universität Hannover, 30167 Hannover, Germany \\ ${ }^{*}$ Corresponding author: patrick.kwee@ aei.mpg.de
}

Received 6 July 2009; accepted 2 September 2009;

posted 15 September 2009 (Doc. ID 113836); published 25 September 2009

\begin{abstract}
We demonstrate an active power stabilization of a Nd:YAG laser employing the optical ac-coupling scheme and derive its fundamental quantum limit. This limit is $3 \mathrm{~dB}$ better than the one encountered in traditional power stabilization schemes. In our experiment, the optical ac coupling improved the shotnoise-limited sensitivity of the stabilization photodetector by a factor of 11.2. With an independent photodetector, we measured a relative power stability of $3.7 \times 10^{-9} \mathrm{~Hz}^{-1 / 2}$ at frequencies of around $200 \mathrm{kHz}$. A detailed investigation of the performance limit of our experiment revealed a novel noise source that disturbed the fundamental mode field in the optical resonator. This effect could be of relevance to many precision experiments using optical resonators. (C) 2009 Optical Society of America
\end{abstract}

OCIS codes: $\quad 140.3425,230.5750,040.5160$.

\section{Introduction}

Active laser power stabilization is important for many optics experiments because free-running laser power fluctuations often limit the sensitivity of these experiments. Interferometric gravitational wave detectors [1-ㅣ] have one of the most demanding power stability requirements. Previous experiments show that traditional power stabilization schemes $[\underline{4}, \underline{5}]$ are barely able to fulfill the power stability requirements for next-generation gravitational wave detectors, especially at low Fourier frequencies $(<100 \mathrm{~Hz})$. The photodetectors used in these experiments are limited at a relative power noise level of $10^{-9} \mathrm{~Hz}^{-1 / 2}$ to $10^{-8} \mathrm{~Hz}^{-1 / 2}$ by unknown noise sources associated with the large photocurrent. Even if these limitations can be overcome, in the future the detected laser power needs to be increased to lower the relative shot noise and to thereby improve the sensitivity of power noise detection. This brings up new problems, such as photodiode saturation and dynamic range limitation of the readout electronics, and requires complicated cooling schemes due to the thermal load in the photo-

0003-6935/09/285423-09\$15.00/0

(C) 2009 Optical Society of America diode [6]. Furthermore, it should be noted that the photodiodes used in current experiments are already operated beyond the maximum manufacturer ratings.

Optical ac coupling [7] increases the sensitivity of a photodetector by using an optical resonator, without increasing the average power on the photodiodes and thus avoids the above-mentioned problems associated with large photocurrents. A power stabilization scheme with optical ac coupling can not only reach a higher stability compared to traditional schemes, but also preserves more light for the actual experiment since less laser light has to be detected.

In this article we present the results of a power stabilization experiment that uses the optical ac-coupling technique. A shot-noise-limited, independently measured relative power stability of $3.7 \times 10^{-9} \mathrm{~Hz}^{-1 / 2}$ at frequencies of around $200 \mathrm{kHz}$ was achieved. This value is consistent with an increased sensitivity of the stabilization photodetector by a factor of 11.2. A power stabilization without optical ac coupling was performed for comparison, which led, as expected, to a worse stability limit of $3.3 \times 10^{-8} \mathrm{~Hz}^{-1 / 2}$.

Our first experiments were limited by resonator internal scattering. A detailed study revealed that 
higher-order transverse modes and the countercirculating fundamental mode in the ring resonator used were excited by scattered light, as already observed in [8]. Our experiment, however, showed that the scattering strength is time dependent and introduces noise in addition to the static finesse reduction studied in [8]. In a series of experiments with improved resonators, we reduced the coupling strength, whereas the fluctuating nature of the effect remained largely unexplained. Simulations verified the adverse effect of scattered light on power stabilization. Furthermore, the coupling of frequency and mode fluctuations and the theoretical limit for power stabilization with optical ac coupling are presented.

The optical ac-coupling stabilization scheme could be used in the future for gravitational wave detectors with long baselines in order to achieve the required power stability, even at low Fourier frequencies of a few Hertz with photodetectors available today.

\section{Theory}

\section{A. Quantum Noise Limit of Different Power Stabilization} Schemes

In the following, three different power stabilization schemes are described. Figure 1 shows the models used to calculate the different quantum noise limits of these schemes. It is assumed that a high-gain feedback control loop is used for power stabilization, which is the case in most experiments. In all three power stabilization schemes, the field $A_{\text {il }}$, called the in-loop field, is used for the control loop sensor in order to compensate power fluctuations of the la-
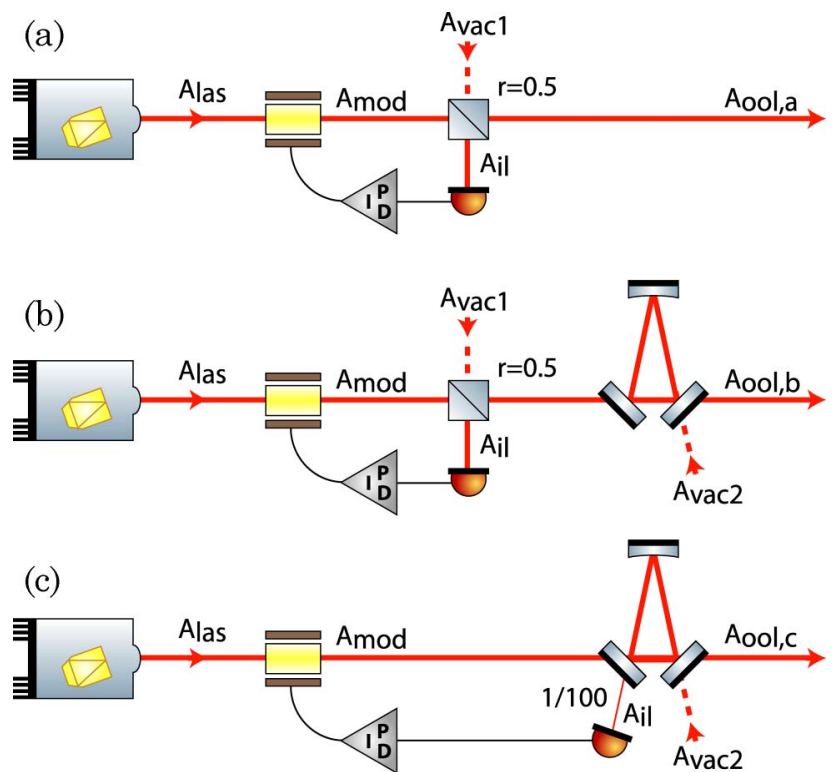

Fig. 1. (Color online) Models used for calculating the quantum noise limit of three different power stabilization schemes: (a) traditional power stabilization, (b) traditional power stabilization with additional resonator in the out-of-loop path, and (c) power stabilization with optical ac coupling. The relative power noise of the outof-loop fields $A_{\text {ool }, a}, A_{\text {ool }, b}$, and $A_{\text {ool, }, c}$ were calculated as functions of the input field $A_{\text {las }}$ and the vacuum fields $A_{\text {vac. }}$. ser field $A_{\text {las }}$ with a power actuator. The fields $A_{\text {ool } a}$, $A_{\text {ool. } b}$, and $A_{\text {ool. } . c}$ are the out-of-loop fields of the three stabilization schemes and are relevant for the actual downstream experiments. The relative power noise of these fields (Fig. 2) was calculated with methods described, e.g., in [9].

To reach the best stability in a traditional scheme [Fig. 1(a)], 50\% of the laser power (beam splitter reflection of $r=0.5$ ) has to be detected on the control loop sensor $A_{\mathrm{il}}$ and $50 \%$ is available in the out-of-loop beam $A_{\text {ool. } . a}[10]$. Because of the feedback control loop, the quantum noise of the field $A_{\mathrm{il}}$ is imprinted on the laser field $A_{\text {mod }}$ and causes power noise at the field $A_{\text {ool. } .}$. The relative power noise of the out-of-loop field is $3 \mathrm{~dB}$ above its quantum noise level. For lower reflectance $(r<0.5)$, the quantum noise of $A_{\mathrm{il}}$ causes an even higher relative power noise of $A_{\text {ool } \alpha}$ and, for higher reflectance $(r>0.5)$, the relative power noise of $A_{\text {ool }, a}$ increases as the absolute power of $A_{\text {ool. } a}$ decreases. Since, for the optimal case $r=0.5$, the outof-loop beam has only $50 \%$ of the original power, the relative power noise of $A_{\text {ool. } a}$ is in total $6 \mathrm{~dB}$ above the quantum noise level of the original beam $A_{\text {las }}$ [Fig. 2(a)].

To improve the power stability in the traditional scheme, a resonator, assumed to be lossless in our model, can be placed in the out-of-loop path [Fig. 1(b)]. A resonator can be seen as a frequency-dependent beam splitter with high transmission at frequencies below the resonator bandwidth and low transmission for higher frequencies. This corresponds to a low-pass filter effect for technical power noise of transmitted fields. Thus, the power noise of $A_{\text {ool }, b}$ is equal to $A_{\text {ool }, a}$ for low frequencies and is dominated by the vacuum fluctuations $A_{\text {vac2 }}$ for frequencies above the resonator bandwidth. As the absolute power of $A_{\text {ool } b}$ is again a factor of 2 smaller than the original beam power, the relative power noise of $A_{\text {ool }, b}$ is $3 \mathrm{~dB}$ above the power noise of $A_{\text {las }}$ for high frequencies [Fig. 2(b)].

In a stabilization scheme with optical ac coupling [Fig. 1(c)] a better power stability can be achieved for frequencies above the ac-coupling resonator (ACC) bandwidth. The transfer function $G(f)$ from relative power fluctuations in front of the ACC to relative power fluctuation in reflection of the resonator was

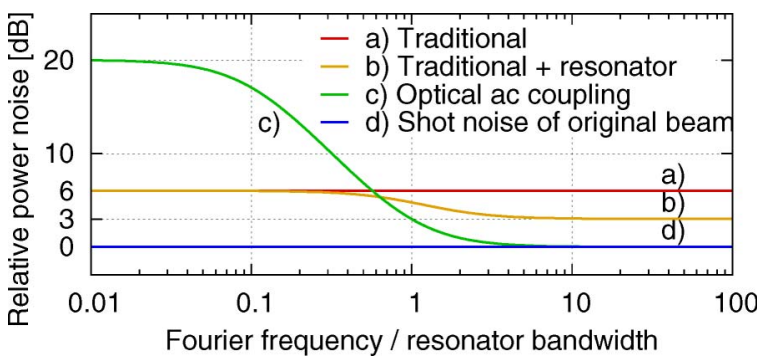

Fig. 2. (Color online) Shot-noise limit for different power stabilization schemes. The limits are given in relation to the relative shot noise of the original beam. Traditional power stabilizations are always 3 to $6 \mathrm{~dB}$ above this reference. A stabilization with optical ac coupling is very close to the relative shot noise of the original beam for Fourier frequencies above the resonator bandwidth. 
derived in [7]. This transfer function, which describes the effect of the optical ac coupling, depends on $f_{0}$, the ACC bandwidth, and $g$, the high-frequency amplification factor $\left(G\left(f \gg f_{0}\right)=g\right)$ :

$$
G(f)=g-(g-1) h(f),
$$

with

$$
h(f)=\frac{1}{1+i f / f_{0}} .
$$

Only the undercoupled case $(g>1)$ can be used in power stabilization, since a phase flip in the overcoupled case would make a power stabilization control loop unstable. An amplification of $g=10$ and a lossless ACC is assumed in our model. The in-loop photodetector is placed in reflection of the ACC and detects $1 \%$ of the original power: $99 \%$ is transmitted through the resonator and is available for the actual experiment. For frequencies far below the ACC bandwidth, the resonator has a high transmission, and the imprinted noise of $A_{\mathrm{il}}$ is dominant at the out-of-loop field $A_{\text {ool.c }}$. The stabilization is, for these frequencies, comparable to a traditional power stabilization with a beam splitter reflection of $r=1 / g^{2}=1 / 100$. The relative power noise of the out-of-loop field $A_{\text {ool, }, c}$ is about $20 \mathrm{~dB}$ (a factor of $g$ ) above the quantum noise of the original beam $A_{\text {las }}$. The reflection of the ACC resonator increases for higher frequencies, causing two effects: on the one hand, the noise imprinted by the control loop onto $A_{\text {mod }}$ is reduced (similar to a traditional power stabilization with large beam splitter reflection $r>0.5$ ). On the other hand, the vacuum fluctuations $A_{\text {vac2 }}$ are mainly reflected by the ACC and dominate the power noise of $A_{\text {ool, } c}$ (similar to the traditional power stabilization with a resonator in the out-of-loop beam). In the end, the relative power noise of $A_{\text {ool,c }}$ is $0.04 \mathrm{~dB}$ (in general $\left[1-1 / g^{2}\right]^{-1 / 2}$ ) above the quantum noise of the original beam [Fig. 2(c)].

To summarize, in a traditional power stabilization, the achievable out-of-loop power noise is $6 \mathrm{~dB}$, with an additional resonator $3 \mathrm{~dB}$, and with optical ac coupling $0.04 \mathrm{~dB}$ (assuming a $g=10 \mathrm{ACC}$ ) above the relative shot noise of the original beam. Associated with this better fundamental limit of the optical ac-coupling technique is the higher available out-of-loop beam power of $99 \%$, compared to $50 \%$ of the original power in the traditional scheme.

The result of this subsection, in combination with the advantages concerning dynamic range issues and photodiode internal noise at large photocurrents, makes the optical ac-coupling power stabilization scheme superior to the traditional schemes for several applications.

\section{B. Noise Couplings}

Because of the optical resonator, power stabilization with optical ac coupling is more sensitive to residual frequency noise, mode fluctuations, and resonator internal scattering. In most experiments, the laser fre- quency and the resonance frequency of the resonator are not stable enough; therefore, an active stabilization of one frequency is necessary. Fluctuations of the remaining difference between laser frequency and resonance frequency of the ACC couple into power fluctuations. For example, due to offset fluctuations of the electronic parts used for frequency stabilization, it is almost inevitable that a small frequency offset $\epsilon$ remains between the two frequencies. The coupling of residual frequency noise to power noise in reflection of the ACC depends on this offset.

Consider the complex field amplitude $U_{\text {in }}$ of a laser beam that is coupled to the ACC. Residual frequency noise can be represented as a phase modulation at a Fourier frequency of $f=\omega / 2 \pi$ with a modulation index of $m \ll 1$ :

$$
\begin{aligned}
U_{\text {in }} & =U_{0} \cdot \exp (i \Omega t+i m \cos (\omega t)) \\
& \approx U_{0} \cdot\left(1+i \frac{m}{2} e^{i \omega t}+i \frac{m}{2} e^{-i \omega t}\right) \cdot e^{i \Omega t},
\end{aligned}
$$

where $\Omega$ is the optical angular frequency. The field inside the resonator $U_{\text {circ }}$ is given by

$$
\begin{aligned}
U_{\text {circ }} \propto & U_{0}\left(h(\epsilon)+i \frac{m}{2} e^{i \omega t} h(f+\epsilon)\right. \\
& \left.+i \frac{m}{2} e^{-i \omega t} h(-f+\epsilon)\right) .
\end{aligned}
$$

The transfer function $N_{F}(f)$ from frequency modulation to relative power modulation in reflection of the resonator can be calculated assuming a small offset $\epsilon \ll f_{0}$ :

$$
N_{F}(f)=\frac{2 \epsilon g^{2}}{f_{0}^{2}} h(f)
$$

The coupling between residual frequency noise and power noise depends on the frequency offset $\epsilon$ and the amplification factor $g$.

Another coupling is caused by mode fluctuations. In this regard, modes can be defined by the polarization state, the different laser frequencies (carrier, sideband frequency), or the spatial intensity distribution of the beam. Even with a constant total power of the incoming beam, a power exchange between resonant and nonresonant modes causes power fluctuations in reflection of the resonator. Such mode fluctuations are produced by, e.g., beam pointing fluctuations, polarization fluctuations, or amplitude fluctuations of modulation sidebands. (Modulation sidebands at frequencies above the resonator bandwidth can be treated as nonresonant modes.)

Consider the following complex field amplitude $U_{\text {in }}$ that is coupled to the ACC. The amplitude of resonant modes can be described by $U_{0}$ and of nonresonant modes by $U_{1}$ such that 


$$
U_{\mathrm{in}}=U_{0} \cdot\left(1-\frac{\left|U_{1}\right|^{2}}{\left|U_{0}\right|^{2}} m e^{i \omega t}\right)+U_{1} \cdot\left(1+m e^{i \omega t}\right) .
$$

Power is exchanged between the two modes with a modulation index $m$ at an angular frequency $\omega$, while, for small $m$, the total power of the beam $\propto$ $\left|U_{\text {in }}\right|^{2}$ stays almost constant.

The transfer function from relative power fluctuations of the nonresonant modes to relative power fluctuations in reflection of the resonator is in the first order given by

$$
N_{M}(f)=\frac{\left|U_{1}\right|^{2}}{\left|U_{0}\right|^{2} / g^{2}+\left|U_{1}\right|^{2}}
$$

This transfer function is independent of the modulation frequency $f$ and depends on the amplification factor $g$ and the power ratio between resonant and nonresonant modes.

Finally, resonator internal scattering can excite higher-order transverse modes or the countercirculating fundamental mode in a ring resonator. This is an additional loss mechanism for the fundamental mode and, thereby, impedance matching and power in reflection of the resonator are changed. A fluctuation of the scattering strength can then cause power fluctuations in the optical ac-coupling scheme.

Transfer functions and power ratios can be simulated using a simple model (Fig. 3). In this model, only two modes of the resonator are considered. One mode is the fundamental mode of the resonator and the second mode is either a higher-order transverse mode or the countercirculating fundamental mode in case a ring resonator is used as the ACC.

The complex field amplitudes inside the resonator are described by a vector

$$
\vec{a}=\left(\begin{array}{c}
a_{0} \\
a_{1}
\end{array}\right)
$$

where $a_{0}$ is the amplitude of the fundamental mode and $a_{1}$ is the amplitude of the second mode. One round trip in the resonator is described by scattering matrices [11]:

$$
\vec{a}^{\prime}=S \cdot P \cdot \vec{a} .
$$

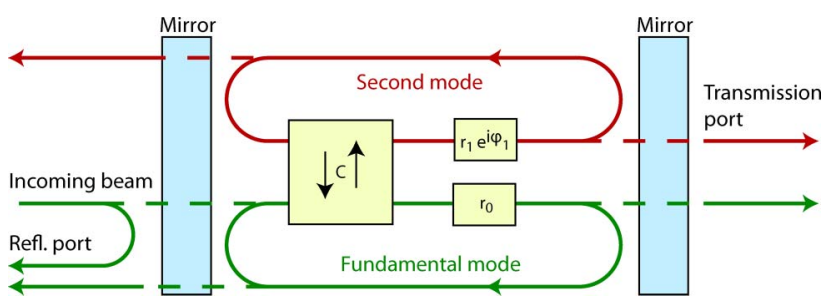

Fig. 3. (Color online) Resonator model used to simulate the complex field amplitudes inside and outside the resonator. To simplify matters, only two possible modes and a coupling between them are considered.
The matrix $P$ describes the individual round trip of the modes, i.e., $r_{i}$ is the reduction factor for the amplitude and $\phi_{i}$ is the phase shift:

$$
P=\left(\begin{array}{cc}
r_{0} e^{i \phi_{0}} & 0 \\
0 & r_{1} e^{i \phi_{1}}
\end{array}\right)
$$

The factors $r_{i}$ include losses due to transmission and absorption at the resonator mirrors and absorption at the resonator internal apertures, which are normally different for a fundamental and a higher-order transverse mode. At the normal operating point of the optical ac-coupling scheme, the fundamental mode is resonant, i.e., $\phi_{0}=0$.

The matrix $S$ describes the coupling of the two modes by scattering. Assuming energy conservation and reciprocal scattering, the matrix depends on only one real parameter, $C$, which describes the coupling between the two modes:

$$
S=\left(\begin{array}{cc}
\sqrt{1-C^{2}} & i C \\
i C & \sqrt{1-C^{2}}
\end{array}\right)
$$

The field amplitudes inside the resonator can now be calculated numerically with this model and, thus, the power at different resonator ports. The simulations that were carried out using this model are described in Section $\underline{4}$.

\section{Methods}

In our experiment (Fig. 4) we used a continuous-wave single-frequency solid-state Nd:YAG nonplanar ring oscillator (NPRO) [12]. This laser had an output power of $2 \mathrm{~W}$ and was operated at a wavelength of $\lambda=1064 \mathrm{~nm}$. The laser had an integrated power stabilization (called "Noise Eater") to suppress the relaxation oscillation at about $1 \mathrm{MHz}$. A resonant electro-optic modulator (EOM) was used to create phase modulation sidebands at $29 \mathrm{MHz}$ for a Pound-Drever-Hall stabilization [13,14].

The laser frequency was controlled with three different actuators. At low Fourier frequencies $(<1 \mathrm{~Hz})$, the temperature of the laser crystal was used as an actuator; for Fourier frequencies up to about $20 \mathrm{kHz}$, the piezoelectric element of the laser crystal was the actuator; and, for Fourier frequencies of up to about $1 \mathrm{MHz}$, a broadband EOM was used as the actuator. The laser beam power was controlled for the downstream experiment with an electro-optic amplitude modulator (EOAM, Thorlabs EO-AM-NR-C2). The EOAM was used to electronically control the polarization and, thereby, the laser power after a subsequent polarizing beam splitter could be changed. With a $\lambda / 4$ and $\lambda / 2$ wave plate in front of the EOAM, the modulation coefficient and the total power downstream of the polarizing beam splitter were adjusted.

An optical three-mirror ring resonator similar to the design described in [15] was used as a mode cleaner (MC) in order to suppress higher-order transverse modes of the beam and to stabilize the beam pointing. A mode matching of better than $99 \%$ to 


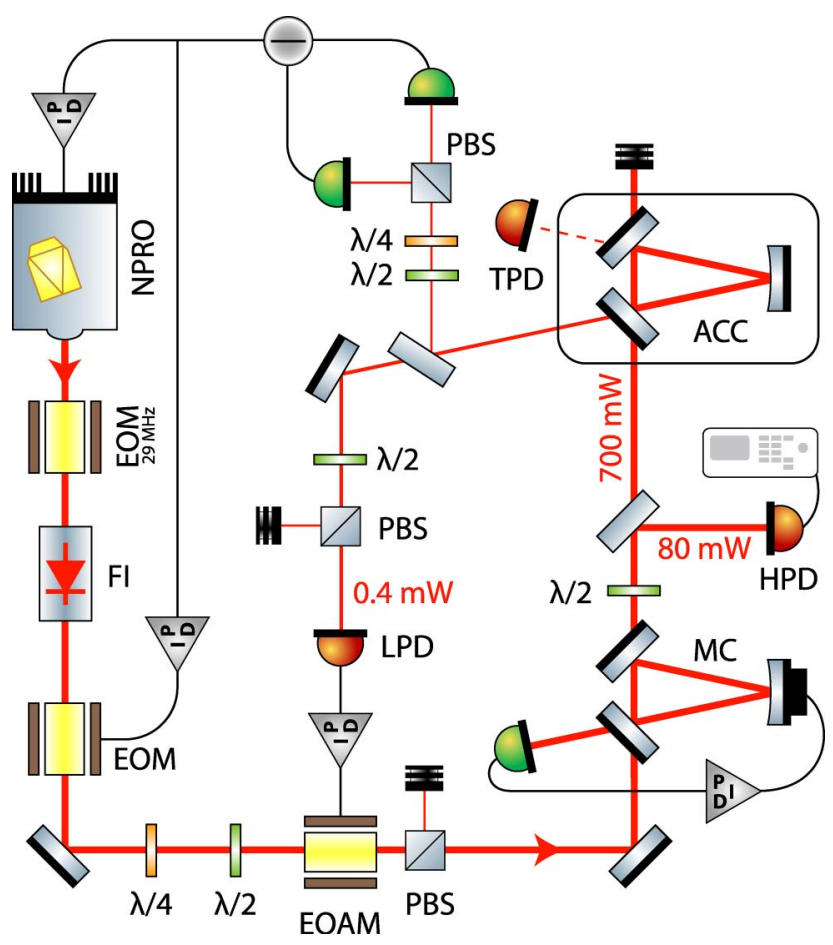

Fig. 4. (Color online) Experimental setup. The laser (NPRO) was filtered by a mode cleaner (MC) and locked to the ac-coupling cavity (ACC). The power noise was measured with detector LPD in reflection of the ACC for the power stabilization control loop. Detector HPD was used as an out-of-loop detector to independently measure the power noise in front of the ACC. Details of the purpose of the other components are given in Section 3. EOM, electrooptic phase modulator; EOAM, electro-optic amplitude modulator; FI, Faraday isolator; PBS, polarizing beam splitter; IPD, servo control filters; $\lambda / 2, \lambda / 4$, wave plates.

the subsequent ACC was only possible thanks to this MC. The MC had a measured finesse of 46 and a bandwidth of $7.8 \mathrm{MHz}$. The round-trip length and, thus, the resonance frequencies of the resonator, were electronically controlled with a piezoelectric element adjusting the position of the curved mirror. The Pound-Drever-Hall method was used to stabilize a fundamental mode of the resonator to the laser frequency. The polarization of the transmitted beam was rotated with a $\lambda / 2$ wave plate such that $98.6 \%$ of the beam was $s$-polarized (vertically).

About $10 \%$ of the beam was sampled and detected with photodetector HPD (Fig. 4). This detector consisted of a $2 \mathrm{~mm}$ InGaAs photodiode (Perkin Elmer C30642) and a transimpedance amplifier $\left(R_{\text {TIA }}=168 \Omega\right)$. A bandwidth of about $20 \mathrm{MHz}$ was achieved, and the electronic noise level was a factor of $\sim 5$ below the shot noise of the detected $60 \mathrm{~mA}$ of photocurrent. This detector was used as an out-ofloop detector for the power noise measurement.

The remaining beam power, of about $680 \mathrm{~mW}$, was directed to the ACC that was used for the optical ac coupling. This resonator had the same geometry as the MC, but had mirrors with different reflectivities. The measured finesse was about 10,000, the bandwidth was $35.7 \mathrm{kHz}$, and the beam waist $w_{0}=$
$372 \mu \mathrm{m}$. The resonator was slightly undercoupled, such that $0.8 \%$ of the incident beam power was reflected due to the impedance mismatch. The transmission of the input and output coupler mirror had a first-order angle dependence of about $-15 \mathrm{ppm} /$ deg with $300 \mathrm{ppm}$ at $45 \mathrm{deg}$ incidence angle. By tilting the curved high-reflective resonator mirror, the incidence angles for the input and output coupler mirrors and, in this way, the impedance matching, were accurately adjusted. All three resonator mirrors and a resonator-internal aperture with a $3 \mathrm{~mm}$ diameter $\left(\approx 4 \times 2 w_{0}\right)$ were glued to an aluminum spacer in order to avoid mechanical resonances of ordinary mirror mounts. The additional losses for the fundamental mode of $10^{-14}$ due to the aperture are negligible compared with the total transmission losses of the mirrors, of about $600 \mathrm{ppm}$. The larger losses for higher-order spatial modes were, however, crucial for this experiment (see Section 4). Without this additional aperture, the smallest aperture, with a diameter of $14 \mathrm{~mm}\left(\approx 19 \times 2 w_{0}\right)$, was given by the spacer itself. The ACC was placed in a pressure-tight aluminum vessel at atmospheric pressure in order to reduce acoustic effects.

The laser frequency was stabilized with the Hänsch-Couillaud method [16] to a fundamental mode resonance of the ACC. A polarizing beam splitter reflected $99.9 \%$ of the $p$-polarized (horizontally) and $\approx 2 \%$ of the $s$-polarized beam for generating the Hänsch-Couillaud error signal. The beam passed a $\lambda / 2$ and $\lambda / 4$ wave plate, and the difference of the $s$ and $p$-polarized power was measured with a polarizing beam splitter and two InGaAs photodiodes. With the additional $\lambda / 2$ wave plate, a difference in the responsivity between the two InGaAs photodiodes was compensated. The feedback control loop had a bandwidth of about $1 \mathrm{MHz}$ and used the three frequency actuators already mentioned.

The remaining power reflected off the ACC was attenuated with a $\lambda / 2$ wave plate and a polarizing beam splitter and was then detected with the photodetector LPD. This detector consisted of a $0.5 \mathrm{~mm}$ InGaAs photodiode (OSI Optoelectronics, FCIInGaAs-500) with a transimpedance amplifier $\left(R_{\text {TIA }}\right.$ $=3 k \Omega)$. A laser power of about $0.4 \mathrm{~mW}(0.3 \mathrm{~mA}$ photocurrent) was detected with a bandwidth of $80 \mathrm{MHz}$. The electronic noise was a factor 3 below the shot noise of the detected photocurrent. This detector was used as an in-loop detector in the power stabilization experiments. The reference current of $0.3 \mathrm{~mA}$ was directly subtracted from the photocurrent. For power stabilization, a bandwidth-optimized low-noise feedback controller and the EOAM were used. The bandwidth of the feedback control loop was about $10 \mathrm{MHz}$ with a loop gain of $>20 \mathrm{~dB}$ for Fourier frequencies below $1 \mathrm{MHz}$. For the design of the controller, the optical ac-coupling transfer function and the MC low pass were taken into account.

The MC was stabilized to the laser frequency, which was stabilized to the resonance frequency of the ACC. The laser power was stabilized with the 
optically ac-coupled detector LPD (in-loop detector) and was independently measured with the detector HPD (out-of-loop detector).

\section{Results}

In our experiment, the out-of-loop measured power stability was first limited by residual frequency noise and resonator internal scattering. However, by carefully controlling the frequency-noise coupling and by suppressing scattered light, the expected power stability could be achieved.

The residual frequency noise between the laser frequency and the resonance frequency of the ACC was between $\approx 0.3 \mathrm{~m} \mathrm{~Hz} \mathrm{~Hz}^{-1 / 2}$ and $\approx 10 \mathrm{~m} \mathrm{~Hz} \mathrm{~Hz}^{-1 / 2}$ for Fourier frequencies between $300 \mathrm{~Hz}$ and $100 \mathrm{kHz}$. As described in Subsection 2.B, the coupling to power fluctuations in reflection of the ACC depended on the offset $\epsilon$. Hence, the frequency offset had to be adjusted to $|\epsilon|<20 \mathrm{~Hz}$ in order for the out-of-loop power stability not to be limited by residual frequency noise.

\section{A. Higher-Order Mode Scattering}

Another effect that limited the power stability in initial experiments was resonator internal scattering. Even though these effects did not have a strong relevance in the final power stability achieved (see Subsection 4.C), they represent novel noise sources in high-finesse cavities so far not described in the literature. As they might be limiting for future resonator experiments, we describe in the following the signature of these effects that we encountered during the course of improving the power stabilization experiment.

Nonstationary scattering between the fundamental mode and higher-order transverse modes or the countercirculating mode caused power fluctuations. Typical out-of-loop measured power stabilities that were limited by scattering are shown in Fig. 5. For frequencies below about $4 \mathrm{kHz}$, the scattering caused a relative power noise of about $10^{-6} \mathrm{~Hz}^{-1 / 2}$ that rolls off toward higher frequencies due to the optical accoupling transfer function.

The excitation of higher-order transverse modes was first observed with an ACC without internal aperture. Dependent on the input power of the ACC, the curvature of the resonator mirrors was slightly changed due to absorption of the Gaussian beam [17]. Thus, the round trip Gouy phase was changed and the resonance frequencies of the higher-order transverse modes were shifted in relation to the fundamental mode. The power on detector LPD was measured as a function of the input power of the ACC while the resonator was locked (Fig. 6). Many different higher-order transverse modes became resonant, indicated by a power increase on detector LPD, while the input power was raised. The power on LPD increased since the impedance matching changed due to a resonant higher-order mode. The coupling between the resonant higher-order mode and the fundamental mode is an additional power loss for the fundamental mode. In addition to this,

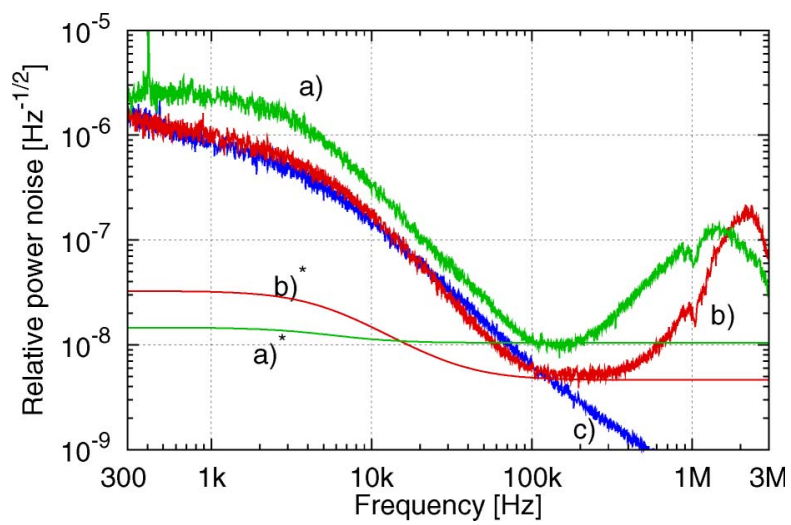

Fig. 5. (Color online) Typical out-of-loop power noise measurements performed with the HPD detector. Scattering to higherorder modes (curve a) or into the countercirculating fundamental mode (curve b) limited the power stability. The expected limits without any scattering are shown as smooth solid curve (curves $a^{*}, b^{*}$ ). For the case of scattering to the countercirculating fundamental mode, a projection of the power noise due to scattering is shown (curve c, see Subsection 4.B).

the field of the higher-order mode leaking out of the resonator into the reflection port cannot destructively interfere with the fundamental mode and, hence, the power on LPD is further increased.

With a charge-coupled device (CCD) camera, the spatial power distribution of the beam in reflection of the ACC was analyzed. Some of the higher-order modes were identified by this measurement (e.g., $\mathrm{TEM}_{1,9}, \mathrm{TEM}_{7.3}, \mathrm{TEM}_{9,1}$, or $\mathrm{TEM}_{20,0}$ ) and we verified that these modes had resonance frequencies close to the fundamental mode.

The coupling between higher-order transverse modes and the fundamental mode, as well as the power increase on detector LPD, was calculated using the two-mode model (see Subsection 2.B). To match the simulation and the experimental results, the coupling factor $C^{2}$ had to be adjusted to a value between $10^{-11}$ and $10^{-10}$ relative power scattering per round trip. The power in higher-order transverse modes depended quadratically on the finesse of the ACC under constant power scattering in our model.

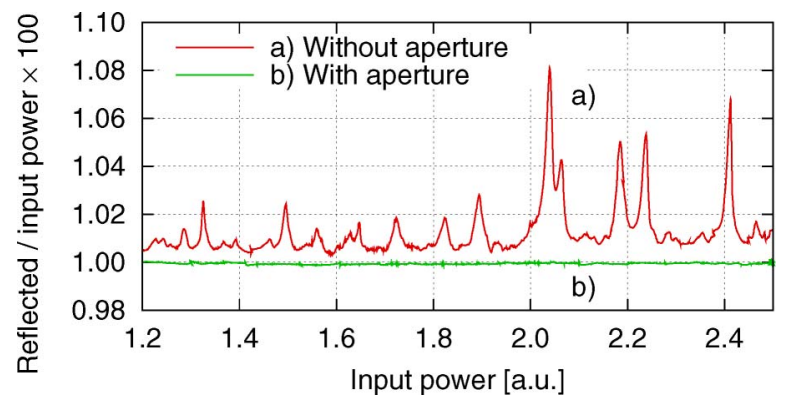

Fig. 6. (Color online) Excitation of higher-order transverse modes by resonator internal scattering. The resonance frequencies of the modes changed with the input power due to a radius of curvature change of the cavity mirrors caused by absorption of a fraction of the circulating power. When a higher-order mode came into resonance, the power in reflection of the ACC was increased. The higher-order modes have been effectively suppressed by a resonator internal aperture. 
This means that this effect becomes increasingly significant for high-finesse resonators.

The out-of-loop power noise was measured with different input power levels. The power noise increased significantly as soon as a higher-order transverse mode became resonant. This effect was not only observed in an optical ac-coupling power stabilization scheme, but also in the transmitted beam even without any power stabilization. (We measured that the free-running relative power noise of the laser of about $2 \times 10^{-7} \mathrm{~Hz}^{-1 / 2}$ was increased to about $1 \times 10^{-6} \mathrm{~Hz}^{-1 / 2}$ up to frequencies of several kilohertz in the transmitted beam of the ACC due to scattering to a higher-order transverse mode. No power stabilization or optical ac coupling was used in this measurement.) The power noise increased when more power was circulating in the higher-order mode. This pointed to the fact that the scattering strength between the fundamental mode and the higher-order mode was fluctuating. This is an important finding, because a fluctuating scattering can not only influence the power of the fundamental mode, but also the phase, which is often used as a reference in metrology and laser stabilization experiments. A hypothesis, namely, that the Gouy phase was fluctuating, was ruled out with this measurement since, in such a case, the power noise had to be maximal at input powers corresponding to the steep slopes in Fig. 6 , which was not the case. The cause of the coupling factor fluctuations is unknown. However, we observed that the scattering and the mode structure in Fig. 6 changed significantly as soon as the curved mirror of the resonator was tilted in steps of only $0.005^{\circ}$ - the smallest controlled steps we could perform. Such a tilt would cause the fundamental mode to move only $\approx 20 \mu \mathrm{m}$ on the curved mirror surface.

The round trip Gouy phase of the ACC was designed in such way that only the resonance frequencies of transverse modes with high mode order were close to the resonance frequencies of the fundamental mode. Because of the installation of a resonator internal aperture, the losses for these higher-order modes have been increased without introducing significant losses for the fundamental mode. Thereby, the excitation of higher-order transverse modes by scattered light was reduced (Fig. 6) and the associated power noise was effectively prevented. Simulations in which the factor $r_{1}$ [Eq. (10)] was reduced to account for the aperture verified these results.

\section{B. Countercirculating Mode Scattering}

The aperture could not avoid an excitation of the countercirculating fundamental mode by scattered light. This was verified with a similar ACC that had a resonator internal aperture but showed a strong scattering into the countercirculating fundamental mode $(100$ to $400 \mu \mathrm{W}$ hitting photodetector TPD at a $680 \mathrm{~mW}$ input power). The cause for the increased scattering is unknown, but might be connected to an outgassing of the epoxy resin used or the o-rings for the vessel. We presume that the scattering occurred at the curved mirror of the resonator since the incidence angle was $3^{\circ}$, much smaller than for the other two mirrors with $44^{\circ}$, and the bidirectional reflectance distribution function (BRDF) normally decreases with increasing scattering angle [18].

The scattering fluctuations caused power fluctuations on the detector LPD in the same way as described in Subsection 4.A (Fig. 5). The power fluctuations of the countercirculating mode have been measured with photodetector TPD. The relative power noise was about $1 \times 10^{-5} \mathrm{~Hz}^{-1 / 2}$ for frequencies below the ACC bandwidth. Numerical simulations were used to calculate the transfer function from power fluctuations on detector TPD to power fluctuations on detector HPD. The two-mode model (see Subsection 2.B) was used, and fluctuations of the coupling factor $C$ were assumed. The power noise on the out-of-loop detector caused by scattering was projected from the measured noise on TPD using this transfer function [Fig. 5(c)]. The absolute level of the transfer function was adjusted to match to the measurement. Furthermore, the transfer function from power noise on detector TPD to detector HPD was measured and agreed with the simulation results. These results represent further evidence for the fluctuating scattering effect.

\section{Power Stabilization Results}

A new ACC that was built using only glues and vessel o-rings having low outgassing showed almost no excitation of the countercirculating fundamental mode $(<15 \mu \mathrm{W}$ hitting photodetector TPD at $680 \mathrm{~mW}$ input power). The optical ac-coupling transfer function from relative power fluctuations at detector HPD to fluctuations at detector LPD was measured. A fit of the model [Eq. (1)] to the data yielded $f_{0}=$ $35.7 \mathrm{kHz}$ and $g=11.2$.

The out-of-loop relative power noise was measured with detector HPD in three different conditions (Fig. 7). First, the free-running power noise of the laser (with Noise Eater) was measured. The relative power noise was about $2 \times 10^{-7} \mathrm{~Hz}^{-1 / 2}$, which is a typical value for these laser models [Fig. 7(a)].

Afterward, the power stabilization control loop was closed [Fig. 7(c)]. The out-of-loop power noise dropped in the whole measurement frequency band by up to $33 \mathrm{~dB}$. The best power stability was $3.7 \times 10^{-9} \mathrm{~Hz}^{-1 / 2}$ at Fourier frequencies of around $200 \mathrm{kHz}$. The expected limit was composed of the shot noise of the in-loop detector (LPD, $0.3 \mathrm{~mA}$ photocurrent, $\left.3.3 \times 10^{-8} \mathrm{~Hz}^{-1 / 2} / G(f)\right)$ and the shot noise of the outof-loop detector (HPD, $60 \mathrm{~mA}$ photocurrent, $2.3 \times$ $10^{-9} \mathrm{~Hz}^{-1 / 2}$ ). The power stability achieved was very close to this limit. For Fourier frequencies between 3 and $20 \mathrm{kHz}$, the power noise measured was about $3 \mathrm{~dB}$ above the expected limit. The source of this excess noise, which is at a level similar to the shot noise of the in-loop detector, is unknown. For Fourier frequencies between $300 \mathrm{~Hz}$ and $1 \mathrm{kHz}$, the power noise was dominated by mechanical resonances of the setup 


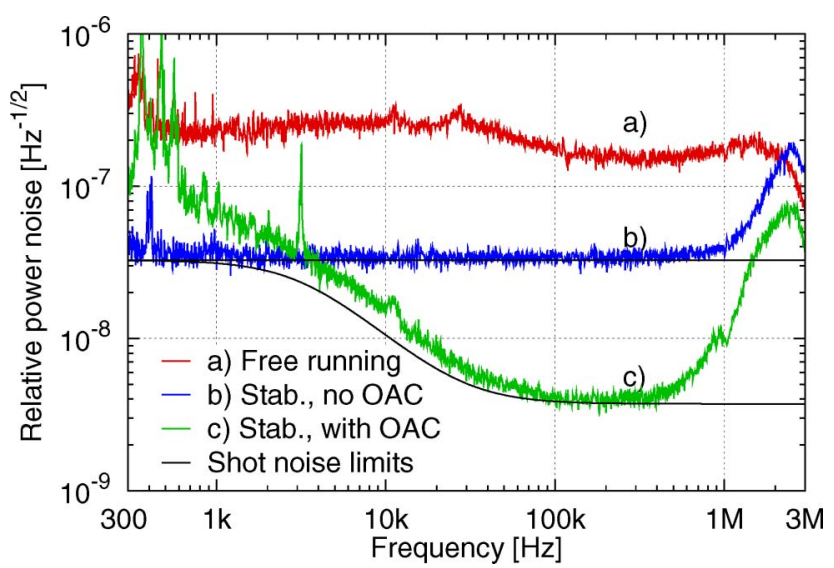

Fig. 7. (Color online) Out-of-loop measured power noise (HPD) using different power stabilization schemes. The results are close to their shot noise limits. With optical ac coupling (OAC) the power stability (curve c) achieved is significantly better than with the equivalent traditional stabilization scheme (curve b).

and, for frequencies above $500 \mathrm{kHz}$, the stabilization was loop-gain limited.

Finally, the laser frequency was not stabilized to the ACC such that the ACC acted as a normal high-reflective mirror. This setup was now equivalent to a traditional power stabilization setup. The power stabilization controller was slightly modified to compensate for the missing amplification $g$, and the attenuation in front of the detector LPD was increased. With unchanged power levels on the in-loop and out-of-loop photodetectors, the power stabilization loop was closed. As expected, the power noise at the out-of-loop detector was limited at a level of the shot noise of the in-loop detector (LPD, $0.3 \mathrm{~mA}$ photocurrent, $3.3 \times 10^{-8} \mathrm{~Hz}^{-1 / 2}$ ) up to Fourier frequencies of about $1 \mathrm{MHz}$ [Fig. 7(b)].

A comparison of the power stabilization with and without optical ac coupling [Figs. 7(b) and 7(c)) clearly shows what we believe is the first experimental proof of the sensitivity improvement of the optical ac-coupling technique. For Fourier frequencies above $4 \mathrm{kHz}$, the out-of-loop power stability was improved by up to a factor of 9 by using the optical ac-coupling scheme.

\section{Discussion}

The feasibility of a power stabilization employing the optical ac-coupling scheme was demonstrated. At Fourier frequencies in the range of the resonator bandwidth, a shot-noise-limited power stability was achieved. Because of the optical ac coupling, the measured residual power noise was up to a factor of 9 below the noise of an equivalent traditional power stabilization. As part of this noise can be attributed to the out-of-loop detector, this result is consistent with an improved shot-noise-limited performance of the stabilization loop by a factor of 11.2.

Experiments with stringent power stability requirements can use this technique to improve the power stability by about 1 order of magnitude. Traditional power stabilization setups that are limited by photo- diode internal noise sources due to high photocurrents can use this stabilization scheme to reduce the power on the photodetector without reducing the shot-noiselimited sensitivity of the photodetectors for frequencies above the resonator bandwidth.

With this scheme, it is theoretically possible to stabilize a laser to its shot noise level with almost no power loss for the control loop sensing. In a traditional scheme, $50 \%$ of the laser power would be lost and the achievable stability would still be a factor of $3 \mathrm{~dB}$ worse.

Furthermore, additional couplings of, e.g., frequency noise or resonator internal scattering need to be taken into account. We have shown clear experimental evidence that a fluctuating scattering process exists in high-finesse resonators. The stationary effect of the scattering was described by a simple model, whereas the fluctuating nature of the scattering remains unexplained. The influence of scattering to higher-order modes can be effectively suppressed by resonator internal apertures. Scattering to the countercirculating fundamental mode can probably be reduced with a resonator geometry with larger incidence angles of the mirrors or can be avoided completely by using a linear resonator. Furthermore, the effects of the resonator internal scattering can probably be reduced by lowering the finesse of the resonator.

One application of the technique demonstrated in this article could be the power stabilization for nextgeneration gravitational wave detectors, such as the Advanced LIGO (Laser Interferometer Gravitational Wave Observatory) [19]. These detectors require very high power stabilities $\left.\approx 2 \times 10^{-9} \mathrm{~Hz}^{-1 / 2}\right)$ at rather low frequencies $(\approx 10 \mathrm{~Hz})$. Such stability levels are problematic to reach with traditional techniques $[4,5]$. The full stability gain of the optical ac-coupling power stabilization scheme is reached only for Fourier frequencies above the resonator bandwidth, although, due to the kilometer-long baseline of such detectors, resonators with very low bandwidth will exist, such as the power recycling cavity with a bandwidth of about $1 \mathrm{~Hz}$.

This research is supported by the Deutsche Forschungsgemeinschaft (DFG) as a part of Sonderforschungsbereich No. 407. The authors thank Albrecht Rüdiger for his helpful comments during the preparation of this manuscript.

\section{References}

1. S. Rowan and J. Hough, "Gravitational wave detection by interferometry (ground and space)," Living Rev. Relativity 3 (2000), http://www.livingreviews.org/lrr-2000-3.

2. K. Somiya, Y. Chen, S. Kawamura, and N. Mio, "Frequency noise and intensity noise of next-generation gravitationalwave detectors with RF/DC readout schemes," Phys. Rev. D 73, 122005 (2006).

3. B. Willke, K. Danzmann, M. Frede, P. King, D. Kracht, P. Kwee, O. Punken, R. Savage, B. Schulz, F. Seifert, C. Veltkamp, S. Wagner, P. Weßels, and L. Winkelmann, "Stabilized high power lasers for advanced gravitational wave detectors," Class. Quantum Grav. 25, 114040 (2008). 
4. F. Seifert, P. Kwee, M. Heurs, B. Willke, and K. Danzmann, "Laser power stabilization for second-generation gravitational wave detectors," Opt. Lett. 31, 2000-2002 (2006).

5. J. Rollins, D. Ottaway, M. Zucker, R. Weiss, and R. Abbott, "Solid-state laser intensity stabilization at the $10^{-8}$ level," Opt. Lett. 29, 1876-1878 (2004).

6. N. Mio, H. Takahashi, and S. Moriwaki, "High-power photo-detection system for next-generation gravitational wave detectors," J. Phys. Conf. Ser. 122, 012014 (2008).

7. P. Kwee, B. Willke, and K. Danzmann, "Optical ac coupling to overcome limitations in the detection of optical power fluctuations," Opt. Lett. 33, 1509-1511 (2008).

8. T. Klaassen, J. de Jong, M. van Exter, and J. Woerdman, "Transverse mode coupling in an optical resonator," Opt. Lett. 30, 1959-1961 (2005).

9. H.-A. Bachor and T. C. Ralph, A Guide to Experiments in Quantum Optics (Wiley-VCH, 2004), Chap. 8.3.

10. M. Taubman, H. Wiseman, D. McClelland, and H. Bachor, "Intensity feedback effects on quantum-limited noise," J. Opt. Soc. Am. B 12, 1792-1800 (1995).

11. A. E. Siegman, Lasers (University Science, 1986), Chap. 11.

12. I. Freitag, A. Tünnermann, and H. Welling, "Power scaling of diode-pumped monolithic Nd:YAG lasers to output powers of several watts," Opt. Commun. 115, 511-515 (1995).
13. E. D. Black, "An introduction to Pound-Drever-Hall laser frequency stabilization," Am. J. Phys. 69, 79-87 (2001).

14. R. W. P. Drever, J. L. Hall, F. V. Kowalski, J. Hough, G. M. Ford, A. J. Munley, and H. Ward, "Laser phase and frequency stabilization using an optical resonator," Appl. Phys. B 31, 97-105 (1983).

15. B. Willke, N. Uehara, E. Gustafson, R. L. Byer, P. J. King, S. U. Seel, and R. L. Savage, "Spatial and temporal filtering of a $10 \mathrm{w}$ Nd:Yag laser with a Fabry-Perot ring-cavity premode cleaner," Opt. Lett. 23, 1704-1706 (1998).

16. T. Hansch and B. Couillaud, "Laser frequency stabilization by polarization spectroscopy of a reflecting reference cavity," Opt. Commun. 35, 441-444 (1980).

17. N. Uehara, E. K. Gustafson, M. M. Fejer, and R. L. Byer, "Modeling of efficient mode-matching and thermal-lensing effect on a laser-beam coupling into a mode-cleaner cavity," Proc. SPIE 2989, 57-68 (1997).

18. T. Klaassen, M. van Exter, and J. Woerdman, "Characterization of scattering in an optical Fabry-Perot resonator," Appl. Opt. 46, 5210-5215 (2007).

19. P. Fritschel, "Second generation instruments for the Laser Interferometer Gravitational Wave Observatory (LIGO)," Proc. SPIE 4856, 282-291 (2003). 